\title{
Head of the Pancreas
}

National Cancer Institute

\section{Source}

National Cancer Institute. Head of the Pancreas. NCI Thesaurus. Code C12269.

That portion of the pancreas lying in the concavity of the duodenum. 